\title{
Research on Application of Virtual Reality Technology in Sports Rehabilitation
}

\author{
Rongzhou Qin \\ Aba Normal University of Sichuan Province, Aba Autonomous Prefecture \\ of Tibetan and Qiang, Sichuan, 623001
}

\begin{abstract}
Virtual reality technology is a comprehensive method of comprehensive computer technology, network technology, simulation technology and humancomputer interaction technology and other new technologies. Through the real simulation of perception channels such as sight, hearing, touch and smell, the user can create a strong feeling and experience of immersive. Based on the virtual reality technology for the rehabilitation of patients brought new vision, combined with the development of virtual reality technology and form of virtual reality technology in patients with abnormal psychological diagnosis and treatment, rehabilitation training and situational teaching of the application, pointed out that virtual reality technology is safe, fun, repeatable and convenient. The future development should focus on reducing R \& D costs, enhancing the immersing experience of participants and enhancing the human-computer interaction, giving play to its flexibility and creativity, etc., for the scientific rehabilitation of patients, fun rehabilitation and maximum rehabilitation to provide more technical support and ideas.
\end{abstract}

Keywords: virtual reality, sports rehabilitation, application research

\section{Introduction}

Virtual Reality (VR) is a brand-new, comprehensive and practical information technology emerging at the end of the 20th century. It is an immersive interactive virtual environment in which the computer produces a set of visual, auditory, olfactory, touch, force, movement and other senses. The operator interacts with 
the human by using necessary interactive, sensing, any angle and virtual threedimensional environment of the object to interact, resulting in the feelings and experience of the virtual environment. Virtual reality technology is a combination of digital image processing, computer graphics, multimedia technology, network technology, sensor and measurement technology, simulation and artificial intelligence and other information technology, thus greatly promoting the development of computer technology. Virtual reality system is to use the advanced computer hardware technology and software tools to design reasonable hardware, software and interactive means to enable participants to interactively observe and manipulate the system-generated virtual world.

Movement disorders refer to the movement is characterized by various obstacles including movement cannot tremor, chorea, torsion spasm, torticollis, dystonia, twitching, twitching and myoclonus and other symptoms. Currently, in the field of rehabilitation of motor disorders, rehabilitation of patients with impaired motor function training is the most important use of VR technology.

In the field of sports rehabilitation, virtual reality technology is the most important use of damaged motor function rehabilitation training. Rehabilitation training aims to enable the body in the shortest possible time through targeted training to eliminate the damage caused by dysfunction. The key factors to determine the effect of rehabilitation training include whether the exercise load of rehabilitation training is moderate, whether the range of exercise is comprehensive, and whether the exercise mode conforms to the daily living habits. VR can provide accurate measurement, auxiliary, monitoring, training and other technologies, so as to fully guarantee the effectiveness of exercise rehabilitation training.

\section{The concept and classification of virtual reality technology}

Virtual reality technology, also known as virtual reality technology, is the use of computer systems, sensory feedback devices and modeling technology can be generated directly applied to the trainer's visual, auditory and tactile sensation, and professional equipment with the help of the human body to stimulate the virtual of the environment or objects for interactive observation and control technology. Virtual reality technology includes three technical components: (1) three-dimensional computer graphics technology; (2) high-definition and high update rate of display technology; (3) multi-function sensor using interactive interface technology. With these special presentation technology and input devices, virtual reality technology allows users to feel immersive.

Compared with traditional rehabilitation equipment, virtual reality system has three important characteristics: immersion, interaction and imagination, emphasizing the virtual reality system in the leading role, so that information processing the system suits the needs of the human body and is consistent with the subjective sense of the person. 
Depending on the presentation mode, VR can be divided into two categories, including immersive virtual reality (such as the use of helmet-style screen or induction gloves) and non-immersive virtual reality (non-immersive virtualreality) Technology, the latter is the image presented on the computer screen or projected onto the screen, the greater the screen, immersed in the virtual reality of the more profound feeling. In addition, the virtual reality system can also be divided according to its size desktop, helmet-type, hole-type, remote type and expansion type.

\section{Virtual reality technology and the role of the mechanism}

Rehabilitation medicine aims to through a variety of means, so that part or all of the function of patients to maximize recovery, in order to obtain the maximum possible self-care, labor and work ability. Although rehabilitation therapy may not completely cure the disease itself, but can improve the symptoms, shorten the course of the disease, reducing the suffering of patients, clinical treatment has become an essential part. The mechanism of elderly rehabilitation therapy involves three key links: repetition, feedback and motivation. According to modern motion control and action learning theory, all human actions are taskoriented characteristics, is the environment or changes in conditions of response. The process of treatment through a different context of meaningful action training and sensory feedback stimulation, and promote system integration, and thus achieve rehabilitation effect. At present, routine exercise therapy and occupational therapy focus too much on disassembling movement exercises and can not provide practical tasks. It is difficult to train the integrated action in real life. The specific performance is that the ability of action acquired in hospital rehabilitation is difficult to adapt. Third, the rehabilitation assessment is to achieve a comprehensive rehabilitation of the premise and foundation, and the existing rehabilitation; The limitation of the equipment makes the traditional rehabilitation treatment still can not achieve the function of quantitative evaluation, rehabilitation and psychological treatment of organic combination, before each treatment, the function of the damaged parts can not be more objective assessment is not conducive to rehabilitation training program The formulation and adjustment.

The advantage of virtual reality technology is that it provides meaningful task training and precise sensory feedback, which can ensure the real and safe training environment of the subject and includes three key training components: repetitive practice, achievement feedback and motivation maintenance. Virtual Reality technology can choose the corresponding rehabilitation training scenario and task-oriented rehabilitation operation according to the patients' mental state and the need of the disease, so as to stimulate and maintain the initiative of the repeated practice of the patients in a variety of feedback forms to reach the rehabilitation effect which can not be realized in the real training. This near-reallife, safer approach to rehabilitation requires more attention and thinking skills that can reflect the problems that may arise when performing complex tasks, 
relieve the fear of stress, and stimulate and train the patient's ability to adapt, Making it easier to return to real life. Numerous studies have shown that patients can learn in the virtual environment, motor skills, and can be applied to the real world real environment. Virtual reality technology can also set up a virtual home of the daily living environment, the patient can be as immersive as the activities, even in the operation errors and the patient's body will not cause injury. The virtual reality technology provides a quantitative assessment of the function of patients before and after rehabilitation training and training to accurately grasp the status of patients with disabilities, residual function and potential capacity is conducive to rehabilitation plane and rehabilitation goals in order to ensure rehabilitation training. Virtual reality provides two types of feedback: real-time feedback of each exercise result and performance feedback after a set of exercises, and timely functional assessment to adjust training plans and training intensity in real-time based on functional improvements, thereby enhancing medical staff and Patients to grasp the therapeutic effect.

In short, compared to traditional rehabilitation training, virtual reality rehabilitation technology closer to the latest concept of rehabilitation therapy, not only to provide real-world similar to the training environment and the task of meaningful needs, but also before and after rehabilitation training in patients with functional status to quantify Assessment, while reducing the intensity of the work of medical personnel.

\section{The existing problems of rehabilitation medicine}

Clinical experience shows that early rehabilitation intervention, the recovery of patients with physical and mental function, prevention of secondary injury and disuse syndrome have played a key role, so rehabilitation and treatment of drugs such as treatment is equally important. China's vast territory, complex terrain, natural disasters in recent years have occurred, to the disaster areas of people's physical and psychological have brought great trauma, coupled with the aging of the increasingly serious problems and changes in disease spectrum, rehabilitation medicine works Faced with unprecedented challenges. Traditional rehabilitation is usually a one-on-one form of physician-patient training, there are many limitations: (1) available resources are not sufficient. Whether a professional rehabilitation physician or rehabilitation equipment is unable to meet the needs of the current rehabilitation of the crowd. (2) training process is monotonous and boring. The traditional training process is usually a repetition of a group of training actions, resulting in the whole process is very boring and it is difficult to effectively mobilize the enthusiasm of patients' active participation, and even make patients have boredom, reducing the efficiency of rehabilitation. (3) quantitative evaluation of the function is difficult to achieve. With the rehabilitation of the continuous progress of the patient's physical condition changes, the amount of activity they bear the intensity is changing, and the current in-patient training in the specific data can not be recorded and preserved, 
is not conducive to rehabilitation training program formulation and adjustment, But also to some extent affected the rehabilitation process.

\section{The scientific principles and its advantages of VR technology being used in sports rehabilitation}

The scientific principle and advantage of the VR technique in rehabilitation is that it provides real-time feedback of each training session and performance feedback after each exercise for patients with exercise rehabilitation training, both of which contribute to the improvement of the patient's Results Awareness. VR technology can not only improve the recreation of exercise through the preparation of virtual environment, but also can stimulate and maintain the motive of repeated practice with various feedbacks. VR technology, fundamentally changed the traditional way of sports rehabilitation, labor and Yat real combination. In this case,

Hemiplegia is the most common sequelae of cerebral infarction, is the side of the limb muscle weakness, activity or adverse activities can not, often accompanied by ipsilateral limb sensory disorders, such as cold and heat do not know, pain, etc., sometimes with There is ipsilateral visual field defect. Rehabilitation medicine is based on kinematics and neurophysiology as the theoretical basis, to take various means of scientific, systematic and comprehensive treatment of patients. Exercise therapy is the most basic treatment in rehabilitation medicine. Exercise therapy has a significant effect on eliminating or alleviating the defects of the patient's function, controlling the abnormality of morbidity and helping the patient to restore the original exercise capacity to the maximum extent.

The experimental results show that VR has the following advantages: First, the virtual environment can make patients feel a sense of being immersed in a natural way to interact with the objects in the virtual environment, thereby enhancing the patient Initiative to participate in the initiative. VR technology can make the virtual environment is very similar to the real environment, the patient can learn in a virtual environment, good skills to move to real life environment. Second, VR technology can not only simplify the rehabilitation of patients with the task, and the virtual coaches than the coaches of the human movement are more consistent. Third, the VR technology can provide feedback in various forms, giving patients the appropriate encouragement, so that the original boring exercise rehabilitation training process becomes easy and interesting. Fourth, VR technology allows users to personalize the settings, the actual situation for the individual patients will be sports training, psychological treatment and functional evaluation of organic combine to develop appropriate exercise rehabilitation training program. 


\section{Conclusion}

VR rehabilitation system can break the limitations of traditional training methods. It can provide different virtual training platform for patients with different movement disorders, so that patients can be rehabilitated in the way of playing games or fulfilling interesting tasks so as to arouse the enthusiasm of patients. The system can also record the training data of the patient in detail. The rehabilitation doctor can monitor the patient's training situation remotely, adjust the training plan and training intensity in real time according to the need, and recommend the rehabilitation treatment scheme. It plays an important role in the rehabilitation of the athletes.

\section{Acknowledgment}

2014 Key Subjects (NO.QXJ1401) of Philosophy and Social Sciences Research Base of Sichuan Province;

2014 Special Fund Project (NO.JY14-02) of Aba Normal University

\section{References}

[1] Li Hongling. Virtual reality technology and its application in rehabilitation medicine. Chinese Journal of Physical Medicine and Rehabilitation, 8 (5), pp.8791,20013

[2] Burdea G,Cioi D, Martin J,et al. Motor retraining in virtual reality: a feasibility stud y for upper -extremity rehabilitation in individuals ith chronic stroke. Journal of Physical Therapy Education, 55(12), pp.68-70, 2011

[3] Sangwoo C,Jeonghun K, Yun K C, et al. Development of virtual real-ity proprioceptive rehabilitation system for stroke patients. Computer Methods and Programs in Biomedicine, 12(10), pp. 18- 20, 2012

[4] Huang Jingyuan, Liu Hongzeng. Preliminary study on the prospect of "virtual reality" rehabilitation project. Journal of Biomedical Engineering, 9(6), pp.58- 61, 2011

[5] Gong Wenqing, Zhu Qiang. Application of virtual reality technology and rehabilitation of movement disorders. Advances in Biomedical Engineering, 8(4), pp.85-87, 2014 